\title{
Sequential interventional treatment of right-sided lung cancer with complete lung atelectasis: A case report
}

\author{
Gang Wu, MD, Zongming Li, MD, Dechao Jiao, MD, and Xinwei Han, MD, Zhengzhou, China
}

\footnotetext{
From the Department of Interventional Radiology, The First Affiliated Hospital of Zhengzhou University, Zhengzhou, China.

Disclosures: Authors have nothing to disclose with regard to commercial support.

Received for publication March 20, 2016; revisions received May 3, 2016; accepted for publication May 4, 2016; available ahead of print June 2, 2016.

Address for reprints: Xinwei Han, MD, Department of Interventional Radiology, The First Affiliated Hospital of Zhengzhou University, Zhengzhou 450052, China (E-mail: 2912123164@qq.com).

J Thorac Cardiovasc Surg 2016;152:e65-7

$0022-5223 / \$ 36.00$

Copyright (c) 2016 by The American Association for Thoracic Surgery

http://dx.doi.org/10.1016/j.jtcvs.2016.05.012
}

Dyspnea as a result of lung cancer and concomitant atelectasis is a serious, acute respiratory condition that can result in death from asphyxiation. Bronchoscopy and biopsy are difficult to perform in dyspneic patients with severe airway stenosis or main bronchus blockage from atelectasis. The timely placement of airway stents can release airway strictures and relieve dyspnea. ${ }^{1}$ We present a case of right lung cancer and atelectasis confirmed by airway imaging and

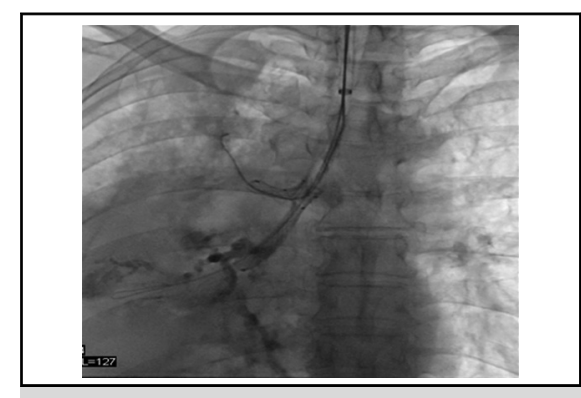

Y-shaped stent placed in right main bronchus, upper bronchus, and intermediate bronchus.

\section{Central Message}

Sequential intervention is an effective and minimally invasive treatment method for lung cancer with complete lung atelectasis.

See Editorial Commentary page e69.

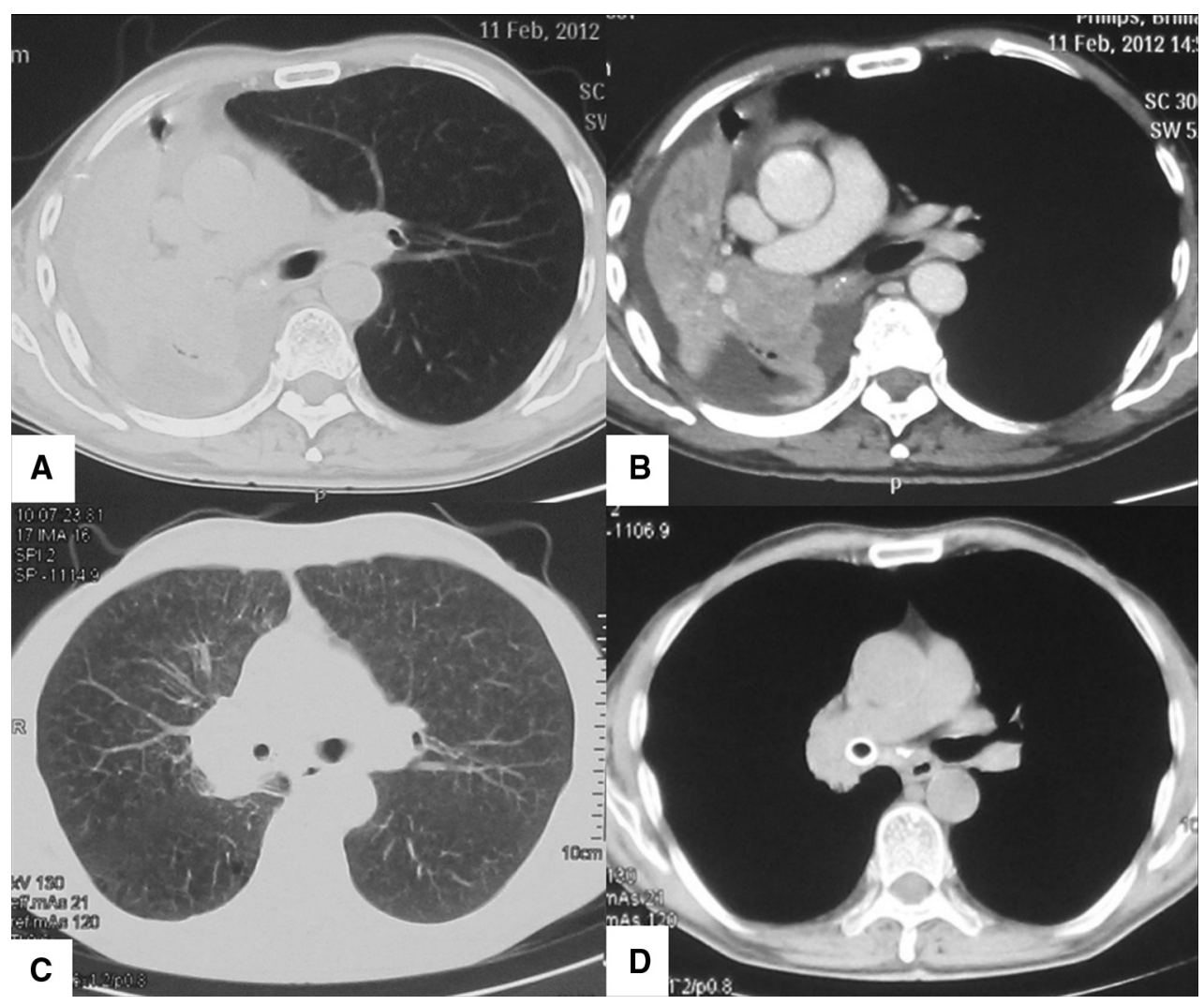

FIGURE 1. Chest computed tomography on admission shows complete atelectasis in the lung window (A) and the mediastinal window (B). Repeated computed tomography in the lung window (C) and the mediastinal window (D) shows a reduction in the size of the tumor in the right lung hilum 1 month after bronchial artery regional perfusion chemotherapy. 


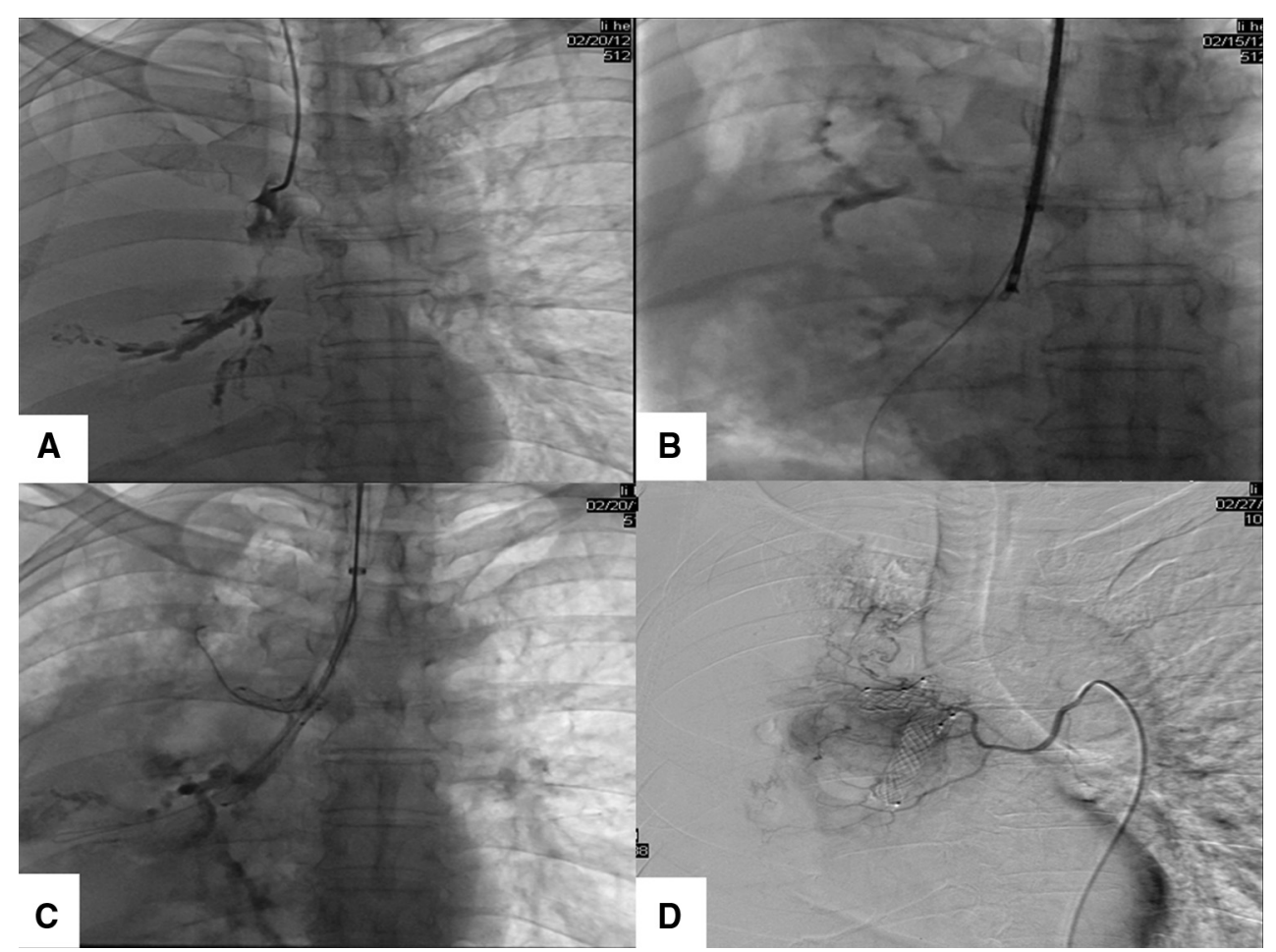

FIGURE 2. A, Bronchography shows complete blockage of the right upper bronchus, the initial segment of the right intermediate bronchus, and the distal right main bronchus. B, Fluoroscopic guidance was used to reach the blocked right bronchus with biopsy forceps inserted through the sheath's canal. C, Fluoroscopy shows release of the Y-shaped stent. D, Right bronchial artery angiography shows thickening of the artery, with abnormally stained areas in the right lung hilum.

interventional biopsy and report its treatment with a Yshaped airway stent to relieve airway blockage, thus relieving the patient's dyspnea.

\section{CLINICAL SUMMARY}

A 66-year-old man came to our department with a 1-month history of productive cough. The patient did not benefit from treatment of his symptoms. Six days before his admission, the patient had become increasingly dyspneic, and chest computed tomography (CT) showed obstruction of the right lung hilum with right lung atelectasis (Figure 1, $A$ and $B$ ). The patient had grade 4 dyspnea, cyanosis, and intermittent fever of $39^{\circ} \mathrm{C}$. A repeated chest $\mathrm{CT}$ scan showed complete right lung atelectasis with plugging of the right main bronchus and a right-shifted mediastinum. The patient was initially treated with oxygen to relieve the dyspnea, but his clinical status worsened.

The patient provided written consent for the use of his medical records for subsequent publication. He underwent bronchography and right bronchial forceps biopsy on day 3 of admission. Bronchography revealed complete blockage of the right upper bronchial opening, the initial part of the right intermediate bronchus, and the distal portion of the right main bronchus (Figure 2, A). Under fluoroscopic guidance, the biopsy forceps were introduced through a sheath, and 3 grain-sized specimens were obtained from different parts of the stenotic area for histopathologic analysis (Figure 2, B), which confirmed the diagnosis of right lung adenocarcinoma. The lung cancer staging was IIIA (T3N2M0).

An appropriate model of a Y-shaped airway stent (Micro-tech Co Ltd, Nanjing, China) was custom made in accordance with the bronchographic and chest CT findings. The right main bronchial portion of the stent measured $16 \mathrm{~mm}$ in diameter and $10 \mathrm{~mm}$ in length, the right upper bronchial part measured $8 \mathrm{~mm}$ in diameter and $12 \mathrm{~mm}$ in length, and the intermediate bronchial portion measured $10 \mathrm{~mm}$ in diameter and $30 \mathrm{~mm}$ in length.

The self-expandable airway stent was implanted 6 days after the patient's admission (Figure 2, C). Repeated transcatheter bronchography showed appropriate stent location and patency of the right bronchial lumen.

Thirteen days after admission, the patient underwent regional perfusion chemotherapy through the right bronchial artery. The angiography showed right bronchial artery thickening with abnormal flaky staining in the right lung 
hilum (Figure 2, D). Chemotherapy, which included $1000 \mathrm{mg}$ fluorouracil, $50 \mathrm{mg}$ pirarubicin, and $60 \mathrm{mg}$ cisplatin, each in $160 \mathrm{~mL}$ of $5 \%$ glucose, was administered slowly through a catheter.

The patient's breathing was normal 1 month after bronchial artery regional perfusion chemotherapy. Repeated chest $\mathrm{CT}$ at this time showed decreased obstruction in the right hilum relative to the preoperative imaging (Figure 1, $C$ and $D$ ). The second chemotherapy was administered 4 weeks later, after which the patient's chest tightness completely resolved. Further follow-up chest CT showed that the tumor continued to decrease in size. Bronchoscopy revealed a small amount of granulation tissue hyperplasia at the stent edges. Radiotherapy was performed 7 days after the second chemotherapy session.

As of this writing, the patient has completed 49 months of follow-up. He has reported feeling subjectively better than before, and at the last follow-up was experiencing only an occasional cough productive of white sputum.

\section{DISCUSSION}

Approximately $80 \%$ of patients with lung cancer have the diagnosis made at an advanced stage, leaving few if any surgical options. ${ }^{2}$ We performed a forceps biopsy with an interventional technique in our patient with severe airway stenosis. This technique allowed us to obtain samples effectively and rapidly for histopathologic analysis with accurate localization, high technical success, and safety. ${ }^{3}$ Simultaneous airway imaging was used to identify specific parts of the tumor that involved the airway, and these data was used to create a unique airway stent. The Y-shaped stent successfully relieved the airway stenosis. ${ }^{4}$ Bronchial artery regional perfusion chemotherapy significantly reduced the tumor's size and further improved the patient's dyspnea. ${ }^{5}$ Finally, radiotherapy was used to destroy any residual neoplastic lesions.

This case validates the use of a series of sequential interventional therapies to treat severe airway stenosis relative to a lung tumor. Not only did this therapy improve the patient's quality of life, it likely also prolonged his survival as a result of shrinkage of the lung adenocarcinoma.

\section{References}

1. Andreetti C, D'Andrilli A, Ibrahim M, Rendina EA. Treatment of a complex tracheobronchial malignant stenosis with a modified conical semicovered selfexpanding stent. J Thorac Cardiovasc Surg. 2013;146:488-9.

2. Videtic GM, Chang JY, Chetty IJ, Ginsburg ME, Kestin LL, Kong FM, et al; Expert Panel on Radiation Oncology-Lung. ACR Appropriateness Criteria early-stage non-small-cell lung cancer. Am J Clin Oncol. 2014; 37:201-7.

3. Li ZM, Wu G, Han XW, Ren KW, Zhu M. Radiology-guided forceps biopsy and airway stenting in severe airway stenosis. Diagn Interv Radiol. 2014;20: $349-52$.

4. Han XW, Wu G, Li YD, Zhang QX, Guan S, Ma N, et al. Overcoming the delivery limitation: results of an approach to implanting an integrated selfexpanding Y-shaped metallic stent in the carina. J Vasc Interv Radiol. 2008; 19:742-7.

5. Nakanishi M, Demura Y, Umeda Y, Mizuno S, Ameshima S, Chiba Y, et al Multi-arterial infusion chemotherapy for non-small cell lung carcinoma-significance of detecting feeding arteries and tumor staining. Lung Cancer. 2008; 61:227-34. 